\title{
Beyond paired quantum Hall states: parafermions and incompressible states in the first excited Landau level
}

\author{
N. Read ${ }^{1,2}$ and E. Rezayi ${ }^{1,3}$ \\ ${ }^{1}$ Institute for Theoretical Physics, University of California, \\ Santa Barbara, California 93106-4030 \\ 2 Departments of Physics and Applied Physics, Yale University, \\ P.O. Box 208120, New Haven, Connecticut 06520-8120 \\ 3 Department of Physics, California State University, \\ Los Angeles, California 90032
}

(February 1, 2008)

\begin{abstract}
The Pfaffian quantum Hall states, which can be viewed as involving pairing either of spinpolarized electrons or of composite fermions, are generalized by finding the exact ground states of certain Hamiltonians with $k+1$-body interactions, for all integers $k \geq 1$. The remarkably simple wavefunctions of these states involve clusters of $k$ particles, and are related to correlators of parafermion currents in two-dimensional conformal field theory. The $k=2$ case is the Pfaffian. For $k \geq 2$, the quasiparticle excitations of these systems are expected to possess nonabelian statistics, like those of the Pfaffian. For $k=3$, these ground states have large overlaps with the ground states of the (2-body) Coulomb-interaction Hamiltonian for electrons in the first excited Landau level at total filling factors $\nu=2+3 / 5,2+2 / 5$.
\end{abstract}

\section{INTRODUCTION}

Trial wavefunctions for fluid states with all the particles in a single Landau level (LL) have played a paradigmatic role in the fractional quantum Hall effect (FQHE) 11] since the work of Laughlin [2]. The Laughlin states were extended to other filling factors using the hierarchy approach [3.4, which can also be used to generate trial wavefunctions, though these are not as elegant or unique as Laughlin's. A different motivation for extensions of the Laughlin wavefunctions to yield other filling factors is the composite fermion approach [5], which generates wavefunctions that are simple, but again not quite unique for each filling factor. In some cases, trial wavefunctions are also energy eigenstates of lowest energy for some Hamiltonian. In the FQHE, these Hamiltonians have usually been found to annihilate the appropriate trial states, and whether this is so can be established without much difficulty. Some early examples are in Refs. [3, 6].

However, these distinct trial wavefunctions at a given filling factor do not necessarily correspond to distinct phases of matter. Phases of matter should be characterized by their ground state quantum numbers (including filling factor or Hall conductance), types of long-range order, ground state degeneracy (if any), and properties of excitations such as whether or not there is an energy gap, and the quantum numbers of the elementary excitations that serve as building blocks for all others. In brief, these characteristics involve universal aspects of the asymptotic low-energy, long-distance physics, and not details of microscopic wavefunctions, either trial ones or exact energy eigenstates of a realistic Hamiltonian. One usually tries to argue that the model Hamiltonian for which the trial ground and some excited states are exact eigenstates yields properties that are characteristic of a phase. If it is true that the energy spectrum has a gap, and hence that the fluids in question are incompressible, then the universal properties should be insensitive to perturbations of the Hamiltonian, and the properties found from the trial states do typify a phase. However, the existence of a gap is hard to establish for the model Hamiltonians, since it involves eigenstates of nonzero energy, which are not known exactly. For the Laughlin state and the pseudopotential Hamiltonian of Haldane [3], the arguments are fairly convincing. (We note that there are also compressible liquids [7, 8], which we believe also to be distinct phases, but for which there are so far no Hamiltonians for which exact eigenstates are known. The same comment applies to crystalline phases.)

In the lowest (or $\mathcal{N}=0$ ) Landau level (LLL) in single-layer systems, the picture that has emerged is that the incompressible fluids are in the phases typified by the Laughlin and hierarchy states, or by the composite fermion approach, these two approaches yielding the same phase for each filling factor with an odd denominator [9, 10]. (There are complications involving spin [11, which we will not discuss here.) In higher LLs, the pseudopotentials [3], that characterize the interactions within the highest partially-filled LL, are different, and the ground states must be reconsidered (the relative importance of the Zeeman energy may also change, but will not be considered in this paper). For example, the quantized Hall plateau (i.e. incompressible fluid) observed at $\nu=5 / 2=2+1 / 2$ [12], where the LLL is filled with electrons of both spin components, and the $1 / 2$ is the filling of the first excited (or $\mathcal{N}=1$ ) LL, does not correspond 
to a hierarchy state; no corresponding plateau is seen at $\nu=1 / 2$ in the LLL. A trial state in which the spins of the electrons in the $\mathcal{N}=1 \mathrm{LL}$ were unpolarized was proposed [6], however, recent work suggests that the true ground state may be polarized [13,14]. Moreover, even for simple fractions such as $2+1 / 3$, the ground state for the Coulomb-interaction values of the pseudopotentials in the $\mathcal{N}=1 \mathrm{LL}$ is on the borderline between compressible and incompressible states, and the Laughlin state does not have such a good overlap with the exact ground state, even when the $V_{1}$ pseudopotential has been increased so as to enter the incompressible region [3].

It is clear that the study of other trial wavefunctions, that are hopefully representative of distinct phases from the hierarchy, could still be of further use in understanding incompressible fluids in higher LLs. One class of these is the paired states, in which one attempts to form Laughlin states of pairs of electrons, in some sense 115,6, 16, 17]. In particular, the so-called Pfaffian state was introduced and related to correlation functions in two-dimensional conformal field theory (CFT) by Moore and Read 16]. This state occurs for filling of the topmost LL of $\nu=1 / q$, for $q$ even for fermions, the physical case. Using this correspondence, they argued that this incompressible fluid phase has fractionally-charged excitations of charge $1 / 2 q$ in electron units, and that these possess nonabelian statistics, a generalization of fractional statistics that we will discuss later. Also, there are neutral fermion excitations; the fluid ground state can be viewed as a Bardeen-Cooper-Schrieffer paired state of these neutral or composite fermions, and the fermion excitations are created by breaking pairs. The Pfaffian ground state is an exact, zero-energy eigenstate of a certain Hamiltonian containing a three-body interaction [17, 18]. The claims about the statistics were reinforced by later work which exhibited the gapless fermion excitations at the edge [19, 20], and the degeneracy of the quasihole states, for the three-body Hamiltonian [21, 18]. In the recent work 13, 14], the true ground state for the Coulomb interaction in the $\mathcal{N}=1 \mathrm{LL}$ was found to have a sizable overlap with the Pfaffian state which can be increased to large values $(97 \%)$ as $V_{1}$ or $V_{3}$ are varied about their Coulombic values. (Morf [13] also found a large overlap with another paired state.) Therefore it is possible that the state observed at $\nu=5 / 2$ is in the phase described by the Pfaffian, as first suggested in Ref. [17].

In this paper we obtain a class of trial wavefunctions by a direct generalization of ideas that are valid for the Pfaffian state, namely the zero-energy eigenstates of a $k+1$-body $\delta$-function interaction, where $k$ is an integer. The existence of the states is established by arguments using operator product expansions (ope's) in CFT [22], and the explicit form of the wavefunctions is obtained and proved to give the ground states. The states involve clusters of $k$ particles, generalizing the pairs in the Pfaffian state. Further analysis gives predictions about the quasi- hole states and nonabelian statistics, which we partially confirm by solving the special Hamiltonians numerically. Then, in Section III we analyse the ground state of the Coulomb interaction in the $\mathcal{N}=1$ LL for $\nu=2+3 / 5$, and compare it with our state at the same filling factor; excellent agreement is found, indicating that our functions are serious contenders to describe the phases in higher LLs, at least in some cases. Further discussion is given in the Conclusion. Some of our results appeared in an earlier unpublished work [23].

\section{SOLUTION OF SPECIAL $K+1$-BODY INTERACTION HAMILTONIANS}

In this Section, we show how to solve certain interaction Hamiltonians, in the sense of finding the zero-energy eigenstates, with the help of operator product expansions (ope's) in conformal field theory (CFT) [22]. We first reinterpret the Pfaffian state in this light, then discuss our Hamiltonians. Then we analyse the quasihole states, and discuss nonabelian statistics and the number of sectors of edge states.

\section{A. Notation and Hamiltonians}

We will first define some notation for a system of particles in the lowest Landau level (LLL) on the sphere [3] (it was used previously in Ref. 18]). The magnetic field is radial and uniform with a total of $N_{\phi}$ flux through the surface, and in the lowest Landau level (LLL) each particle has orbital angular momentum $N_{\phi} / 2$. The LLL wave functions on a sphere are usually written (in a certain gauge [3]) in terms of "spinor" (or "homogeneous") coordinates $u_{i}$ and $v_{i}$ for each particle $i=1, \ldots, N$, with $u_{i}=e^{i \phi_{i} / 2} \cos \theta_{i} / 2, v_{i}=e^{-i \phi_{i} / 2} \sin \theta_{i} / 2$, where $\theta_{i}, \phi_{i}$ are the spherical polar coordinates on the sphere. Since these imply that $u_{i}, v_{i}$ are not independent complex numbers, it is often more convenient, and will simplify the writing, to use a nonredundant parametrization of the sphere by a single complex variable. This is done by stereographic projection, which gives the definition $z_{i}=2 R v_{i} / u_{i}$, where $R$ is the radius of the sphere. Single-particle basis states in the LLL then take the form $z_{i}^{m} /\left(1+\left|z_{i}\right|^{2} / 4 R^{2}\right)^{1+N_{\phi} / 2}$, where the $L_{z}$ angular momentum quantum number is $L_{z}=N_{\phi} / 2-m$, so $m \leq N_{\phi}$. Many-particle states can thus be written as

$$
\Psi=\tilde{\Psi} \prod_{i}\left(1+\left|z_{i}\right|^{2} / 4 R^{2}\right)^{-\left(1+N_{\phi} / 2\right)}
$$

and $\tilde{\Psi}$ must be a polynomial of degree no higher than $N_{\phi}$ in each $z_{i}$. Therefore, in the following we need specify only $\tilde{\Psi}$ in order to describe a state. The function $\tilde{\Psi}$ for a ground state on the sphere can also be used 
to construct a wavefunction suitable for a corresponding disk-shaped ground state on the plane, by multiplying by $\exp \left(-\frac{1}{4} \sum_{i}\left|z_{i}\right|^{2}\right)$.

The Pfaffian state is defined as [16]

$$
\tilde{\Psi}_{\mathrm{Pf}}\left(z_{1}, \ldots, z_{N}\right)=\operatorname{Pf}\left(\frac{1}{z_{i}-z_{j}}\right) \prod_{i<j}\left(z_{i}-z_{j}\right)^{q} .
$$

The Pfaffian

$$
\operatorname{Pf} M_{i j}=\left(2^{N / 2}(N / 2) !\right)^{-1} \sum_{P} \operatorname{sgn} P \prod_{r=1}^{N / 2} M_{P(2 r-1) P(2 r)}
$$

of an antisymmetric $N \times N$ matrix $M(N$ even) is the antisymmetrized sum over all pairings $\left(z_{i}-z_{j}\right)^{-1}$ (the analogous pairing in the spin-singlet case appears in the Haldane-Rezayi state [6]). The filling factor of the state is $\nu=N / N_{\phi} \rightarrow 1 / q$ as $N \rightarrow \infty$ (since $N_{\phi}=q(N-1)-1$ ), and $q$ is odd for a boson state and even for fermions.

The Pfaffian state for $q=1, q=2$ is the ground state of a three-body Hamiltonian [17, 18]. For these cases, the Hamiltonian penalises the closest approach of any three particles allowed by the statistics. Thus for $q=1$, where the particles are bosons (note that we reserve the term "particles" for the underlying particles, which are either charged bosons or charged fermions [electrons], and not for composite particles), the Hamiltonian can be taken to be 17

$$
H=V \sum_{i<j<k} \delta^{2}\left(z_{i}-z_{j}\right) \delta^{2}\left(z_{i}-z_{k}\right),
$$

where the sum is over distinct triples of particles [24]. For numerical purposes on the sphere, it is more convenient to work with a projection operator form of the threebody Hamiltonian, instead of the $\delta$-functions in (2.4). The closest approach of three particles on the sphere corresponds to the state of maximum possible total angular momentum for the three. If the particles are bosons, the largest possible total angular momentum is $3 N_{\phi} / 2$ (recall that each particle has angular momentum $N_{\phi} / 2$ ). Then, for the $q=1$ case, the Hamiltonian may be taken as proportional to the projection operator onto the (unique) multiplet of maximum angular momentum for each triple of bosons:

$$
H=\sum_{i<j<k} V P_{i j k}\left(3 N_{\phi} / 2\right) .
$$

The same trick works for the three-body interaction of fermions giving the $q=2$ case; in this case, the maximum total angular momentum of three particles is $3 N_{\phi} / 2-3$. On the plane, the latter Hamiltonian corresponds to derivatives of delta functions. For these two cases, the Pfaffian state on the sphere is the unique energy eigenstate of zero energy at the stated $N_{\phi}$ value. (We will refer to such states as zero-energy states hereafter.) For larger $q$, these Hamiltonians can be generalized, in such a way that the zero-energy states are obtained from those for $q=1$ by multiplying by $\prod\left(z_{i}-z_{j}\right)^{q-1}$ (it is assumed that for $q$ odd, we are discussing bosons, and for $q$ even, fermions). The presence of the latter factor implies that they are all zero-energy eigenstates of the projection operators for any two particles onto relative angular momentum $M=0,2, \ldots, q-3$ ( $q$ odd), or $M=1,3$, $\ldots, q-3$ ( $q$ even) [or the corresponding total angular momenta $N_{\phi}, N_{\phi}-2, \ldots, N_{\phi}-q+3,(q$ odd), etc. $]$. The space of states annihilated by such projections is in one-to-one correspondence with the full space of states of the $q=1$ case, and the desired three-body projection operator [onto angular momentum $3 N_{\phi} / 2-3(q-1)$ ] is the unique one that corresponds under this mapping to that already mentioned for $q=1$. For each $q$, the Hamiltonian can then be taken to be the sum of the three-body and all of these two-body projection operators. A very similar approach works for the other Hamiltonians studied in this paper, so that results for higher $\nu^{-1}$ can be deduced easily from those for the minimal $\nu^{-1}$ for each type of state. These Hamiltonians can also be written in terms of $\delta$-functions and their derivatives, so as to arrive at a form suitable for use in geometries other than the sphere.

The goal of this paper can now be stated: we wish to generalise the solution for the zero-energy states of the three-body Hamiltonian (2.4) or (2.5) to $k+1$-body Hamiltonians of the same closest approach form, for every $k$ (note that for $k=1$, we can consider the solutions to be the Laughlin ground and quasihole states [3]). We will demonstrate the existence of an elegant solution to this problem by using conformal field theory, construct the ground state wavefunctions explicitly, and then compare the states with solutions for the Coulomb interaction in the second LL.

\section{B. Ope's and the Pfaffian state}

First we will reconsider the Pfaffian state and show how its property of being the unique zero-energy state for a certain Hamiltonian, that is of vanishing as three particles come to the same point, is related to operator product expansions in the corresponding CFT. We then generalize this to find the solutions to the simplest examples of $k+1$-body Hamiltonians, for each $k$. The use of CFT leads to an existence proof for these wavefunctions, and in principle determines the wavefunctions uniquely.

The basic idea of Ref. [16] is that the wavefunction of our 2 space-, 1 time-dimensional system can be related to a correlator of a certain conformal field theory. Here we are not interested in the simple Laughlin-Jastrow factors, but in the other parts that may produce nonabelian 
statistics. For the Pfaffian case, this part is the theory of free massless Majorana Fermi fields in 2 Euclidean spacetime dimensions 16:

$$
\tilde{\Psi}_{\mathrm{Pf}}=\left\langle\psi\left(z_{1}\right) \cdots \psi\left(z_{N}\right)\right\rangle \prod_{i<j}\left(z_{i}-z_{j}\right)^{q}
$$

in which the correlator can be evaluated using Wick's theorem and $\langle\psi(z) \psi(w)\rangle=-\langle\psi(w) \psi(z)\rangle=(z-w)^{-1}$. The Fermi fields can also be characterized by the operator product expansions (ope's) which hold inside correlators 22

$$
\begin{aligned}
\psi(z) \psi(w) & \sim(z-w)^{-1}\left[I+2(z-w)^{2} T(w)+\ldots\right], \\
\psi(z) I(w) & \sim \psi(w)+(z-w) \partial \psi(w)+\ldots,
\end{aligned}
$$

as $z \rightarrow w$, where $I$ is the identity (which is a constant), and $T(z)=-\frac{1}{2} \psi \partial \psi$ is the stress tensor. Throughout, $\partial \phi(z)$ will mean $\partial \phi(z) / \partial z$ and dots ... will denote higher order, less-singular terms. While the correlator of Majorana fermions can be found by Wick's theorem, the use of ope's is more general and can be applied in a vast number of situations where there is no simple Wick's theorem.

We now show how the ope for the fermions guarantees that the state is a zero-energy eigenstate for the threebody interaction of Greiter, Wen and Wilczek [17]. This can be seen directly 17 from the explicit wavefunction (2.2), however, the ope's provide a general argument that can be used even when the wavefunctions are unknown, if the function is a correlator of fields with known ope's. Suppose that in the correlator (without loss of generality) first $z_{2} \rightarrow z_{1}$, then $z_{3} \rightarrow z_{1}$, and take the most singular term of each product. The first limit (using Eq. (2.7)) gives $\left(z_{1}-z_{2}\right)^{-1} I$, and the second (using Eq. (2.8)) then gives $\left(z_{2}-z_{1}\right)^{-1} \psi\left(z_{1}\right)$. Multiplying by the LaughlinJastrow factor $\tilde{\Psi}_{\mathrm{LJ}}=\prod_{i<j}\left(z_{i}-z_{j}\right)$ we find that $\tilde{\Psi}_{\mathrm{Pf}}$ for $q=1$ vanishes as $\left(z_{3}-z_{1}\right)\left(z_{3}-z_{2}\right)$, i.e. quadratically. Hence for the short-range interaction among bosons in Eq. (2.4) we obtain zero energy [17. Furthermore it is the densest such state [17,20,18]. The same argument applies for $q>1$ by construction.

\section{Parafermion states}

We next generalize the ideas to solve Hamiltonians with $k+1$-body interactions. The simplest such Hamiltonian, which will give the highest density such state for each $k$, which is always a state for bosons, is a $\delta$-function interaction between the $k+1$ particles:

$$
H=V \sum_{i_{1}<i_{2}<\cdots, i_{k+1}} \delta^{2}\left(z_{i_{1}}-z_{i_{2}}\right) \delta^{2}\left(z_{i_{2}}-z_{i_{3}}\right) \cdots \delta^{2}\left(z_{i_{k}}-z_{i_{k+1}}\right) .
$$

As we will see, the wavefunctions involve dividing the particles into clusters of $k$ each. To generalize the approach just described for the Pfaffian, we will consider a natural generalization of the ope algebra of Majorana fermions, which is the algebra of $\mathbf{Z}_{k}$ parafermions [25]. This consists of a set of fields $\psi_{\ell}(z), \ell=1, \ldots, k-1$ with ope's $\left(\psi_{\ell}^{\dagger}(z)=\psi_{k-\ell}(z)\right)$

$$
\begin{aligned}
\psi_{\ell}(z) \psi_{\ell^{\prime}}\left(z^{\prime}\right) & \sim c_{\ell, \ell^{\prime}}\left(z-z^{\prime}\right)^{-\left(\Delta_{\ell}+\Delta_{\ell^{\prime}}-\Delta_{\ell+\ell^{\prime}}\right)}\left[\psi_{\ell+\ell^{\prime}}\left(z^{\prime}\right)+\ldots\right] \quad\left(\ell+\ell^{\prime}<k\right), \\
\psi_{\ell}(z) \psi_{\ell^{\prime}}^{\dagger}\left(z^{\prime}\right) & \sim c_{\ell, k-\ell^{\prime}}\left(z-z^{\prime}\right)^{-\left(\Delta_{\ell}+\Delta_{\ell^{\prime}}-\Delta_{\ell-\ell^{\prime}}\right)}\left[\psi_{\ell-\ell^{\prime}}\left(z^{\prime}\right)+\ldots\right] \quad\left(\ell^{\prime}<\ell\right), \\
\psi_{\ell}(z) \psi_{\ell}^{\dagger}\left(z^{\prime}\right) & \sim\left(z-z^{\prime}\right)^{-2 \Delta_{\ell}}\left[I+\frac{2 \Delta_{\ell}}{c}\left(z-z^{\prime}\right)^{2} T\left(z^{\prime}\right)+\ldots\right], \\
T(z) \psi_{\ell}\left(z^{\prime}\right) & \sim \frac{\Delta_{\ell}}{\left(z-z^{\prime}\right)^{2}} \psi_{\ell}\left(z^{\prime}\right)+\frac{1}{z-z^{\prime}} \partial \psi_{\ell}\left(z^{\prime}\right)+\ldots
\end{aligned}
$$

The consistency of the algebra puts conditions on the conformal weights $\Delta_{\ell}$ of the $\psi_{\ell}$ 's. The simplest solution to the conditions is $\Delta_{\ell}=\ell(k-\ell) / k$, and with this choice the values of the central charge $c=2(k-1) /(k+2)$ and the numerical coefficients $c_{\ell, \ell^{\prime}}$ are then uniquely determined by consistency. With these choices, we obtain the algebra usually known as $\mathbf{Z}_{k}$ parafermions. (Other solutions for the $\Delta_{\ell}$ are listed in Ref. [25], and some are studied further. In these cases the other parameters are not uniquely determined. These other algebras will not be needed here, but may be relevant in other FQHE problems. The procedure below will generate wavefunctions in all cases, but these may not always be solutions to simple Hamiltonians.) For $k=2, \psi_{1}=\psi$ is a Majorana fermion, and the ope's are the same as Eqs. (2.7,2.8) above.

Consider the function

$$
\tilde{\Psi}_{\text {para }}^{(m)}\left(z_{1}, \ldots, z_{N}\right)=\left\langle\psi_{1}\left(z_{1}\right) \cdots \psi_{1}\left(z_{N}\right)\right\rangle \tilde{\Psi}_{\mathrm{LJ}}^{M+2 / k}
$$

where $N$ is divisible by $k$ and $M \geq 0$ is an integer. The $\tilde{\Psi}_{\mathrm{LJ}}^{M+2 / k}$ factor renders (2.14) nonsingular $\sim\left(z_{i}-z_{j}\right)^{M}$ as any $z_{i} \rightarrow z_{j}$, according to Eq. (2.10) with $\Delta_{\ell}+\Delta_{\ell^{\prime}}-\Delta_{\ell+\ell^{\prime}}=2 \ell \ell^{\prime} / k$, which gives $2 / k$ for $\ell=\ell^{\prime}=1$. $\tilde{\Psi}_{\text {para }}$ is totally symmetric for $M$ even, antisymmetric for $M$ odd, so describes bosons or fermions, respectively. As any $z_{i} \rightarrow \infty$, the correlator $\sim z_{i}^{-2 \Delta_{1}}$, because the separations among the other $N-1 \psi_{1}$ 's are then relatively small, and the ope's 
imply that they "fuse" to form a single $\psi_{1}^{\dagger}$. Hence $\tilde{\Psi}_{\text {para }}$ is a polynomial of degree $N_{\phi}=\nu^{-1} N-(M+2)$, where $\nu=k /(M k+2)$ is the filling factor.

Taking now the $M=0$ case, we can show that the function vanishes quadratically as any $k+1$ particles come to the same point, say $z_{2}, \ldots, z_{k+1}$ approach $z_{1}$ one by one, by a similar argument based on the ope's as for the Pfaffian. As the first $k-1$ particles approach $z_{1}$, the most singular (or most slowly vanishing) terms must give the same result as the $N=k$ case of $\tilde{\Psi}_{\text {para }}$, which is a constant [25]; one additional particle gives $\psi_{1}\left(z_{k+1}\right) I \sim \psi_{1}\left(z_{1}\right)$, and the LJ factors give $\left(z_{k+1}-z_{1}\right)^{2}$. Hence like the Pfaffian state with $q=M+1=1$, this state is a zero-energy state for the $k+1$-body $\delta$-function interaction of bosons, and this fact again extends to $M>0$. This shows that the desired wavefunctions exist, provided the CFT correlators do; but the parafermion theories are well-studied and can be constructed from other well-understood theories [25[26], so correlators with the properties specified by the ope's do exist. Notice that, had we chosen to use a different parafermion operator $\psi_{\ell}(\ell \leq k / 2)$ in place of all the $\psi_{1}$ 's, we would have obtained a polynomial that vanishes more rapidly when $k+1 z_{i}$ 's coincide, and a lower filling factor.

Explicit functions can be obtained using the idea of grouping particles into clusters of $k$ [15]. The following procedure gives a symmetric $\tilde{\Psi}_{\text {para }}^{(0)}$ of the correct degree which is the Laughlin state for $k=1$, and the Pfaffian state for $k=2$ : Divide the particles into clusters of $k$. For each pair of distinct groups, say $z_{1}, \ldots, z_{k}$ and $z_{k+1}, \ldots, z_{2 k}$, we define factors $\chi$ by (for $k \geq 2$ )

$$
\chi\left(z_{1}, \ldots, z_{k} ; z_{k+1}, \ldots, z_{2 k}\right)=\left(z_{1}-z_{k+1}\right)\left(z_{1}-z_{k+2}\right)\left(z_{2}-z_{k+2}\right)\left(z_{2}-z_{k+3}\right) \cdots\left(z_{k}-z_{2 k}\right)\left(z_{k}-z_{k+1}\right),
$$

that is, each member of a cluster is connected with only two members of the other cluster, by a factor $z_{i}-z_{j}$. For $k=1$, we would have $\chi=\left(z_{1}-z_{2}\right)^{2}$. Now we multiply these for all distinct pairs of clusters, and symmetrize the whole expression, to obtain

$$
\tilde{\Psi}_{\mathrm{para}}^{(0)}=\sum_{P \in S_{N}}^{\prime} \prod_{0 \leq r<s<N / k} \chi\left(z_{P(k r+1)}, \ldots, z_{P(k(r+1))} ; z_{P(k s+1)}, \ldots, z_{P(k(s+1))}\right)
$$

where $S_{N}$ is the permutation group on $N$ objects, and the prime on the sum denotes that the summation can be restricted to permutations obeying $P(1)<P(k)<$ $\cdots<P(N-k+1)$, which eliminates redundant permutations of the clusters; otherwise each term would appear $(N / k)$ ! times (in the expression for the Pfaffian above, we instead summed over all permutations but divided by the number of times each distinct product appeared). Hence this function is totally symmetric, even with this restriction. To obtain larger $M>0$, we must multiply by $\tilde{\Psi}_{\mathrm{LJ}}^{M}$. The degree of the function in each $z_{i}$ is then $N_{\phi}=M(N-1)+2(N / k-1)=\nu^{-1} N-(M+2)$, as for the parafermion correlator times LJ factor above.

Next we show that our function (for $M=0$ ) vanishes quadratically whenever any $k+1$ particles come to the same point. It is sufficient to consider each term in the sum over permutations separately. Clearly, we must then consider many possibilities, in which the $k+1$ particles are distributed among different clusters, ranging from all but one in the same cluster, to each in distinct clusters. We will organise our proof in the following way: first we show that each term vanishes if $k$ particles come to the same point, except in the case of all $k$ in the same cluster. Then we use the evident fact that for $k$ particles in one cluster, one in another, the term vanishes (this follows directly from the definition of $\chi$ itself).

We must consider $k$ distinct $z_{i}$ 's, chosen in any way from up to $k$ distinct clusters. We may label these by the corresponding position in a cluster (from 1 to $k$ ), and the label (like $r, s$ ) for the cluster, that appear inside the permutation operator $P$. These determine when the product of $\chi$ 's vanishes. Put another way, it is sufficient to consider the term where $P$ is the identity. Now consider the values for the position within a cluster as arranged on a circle (or clock face), with the numbers increasing clockwise until $k$ is reached, which is followed by 1 , so that 1 is adjacent to 2 and $k, 2$ is adjacent to 1 and 3 , and so on. The $\chi$ 's containing any two selected $z_{i}$ 's vanish only in two cases: (i) if members of distinct clusters occur at the same place on the clock; (ii) if members of distinct clusters occur at adjacent positions, but only if the position of the later cluster is arrived at by moving clockwise from the earlier by one step (see the definition of $\chi$ ). We can then describe the structure of the term containing any set of $k z_{i}$ 's by labelling positions on the clock face with the numbers of the clusters in which the $z_{i}$ 's appear. Members of the same cluster must be at different clock positions because they are distinct $z_{i}$ 's. Clearly, if a term is to have any possibility of not vanishing, we must choose all the clock positions to be distinct. We then have a single number of a cluster assigned to each clock position. We will now show that it is not possible to arrange these in such a way that the term does not vanish, except by choosing all the $z_{i}$ 's from a single cluster. We can view the clock as divided into regions (possibly consisting of positions that are not all adjacent) that have been labelled (or "colored") with a single cluster number. To avoid a vanishing factor, the numbers of the clusters 
must decrease as one moves clockwise from one region to another. But the clock is not simply connected, and so this cannot be done all the way around the clock, unless there is only a single region (or cluster) involved. In the latter case, consideration of any one other $z_{i}$ shows that the term vanishes, as mentioned before. Therefore, we have shown that the function vanishes at least linearly, but since it is totally symmetric after summing over permutations, it will actually vanish quadratically as any $k+1$ particles come to the same point.

We have shown that this vanishing property follows from the parafermion ope's, which should determine the function, however the vanishing property alone may be only a necessary and not a sufficient condition to determine that the function is the parafermion correlator times $\tilde{\Psi}_{\mathrm{LJ}}^{M}$. Nonetheless, since we have demonstrated it for an explicit function, and since this function seems to be unique, on the basis at least of numerical verification for $k=3,4$ (discussed further below), we conjecture that the parafermion correlators for all $k$ are given by this construction (by dividing $\tilde{\Psi}_{\text {para }}^{(0)}$ by $\tilde{\Psi}_{\mathrm{LJ}}^{2 / k}$ ). It should be possible to prove or disprove this statement by studying the properties of our function as various combinations of $z_{i}$ 's coincide, and comparing these with the ope's. This was done for various paired states in Ref. [27], but we will not consider it further.

\section{Quasihole states and nonabelian statistics}

In this Subsection, we argue that states containing quasiholes (carrying charge $1 /(M k+2)$ each) for the parafermion states with $k>2$ can be constructed in analogy with the Pfaffian case, and that nonabelian statistics are expected in all cases. The arguments go as follows. We will again begin with the Pfaffian state.

The two-quasihole wavefunction proposed in Ref. [16] was

$$
\tilde{\Psi}_{\mathrm{Pf}+2 \mathrm{qh}}\left(z_{1}, \ldots ; w_{1}, w_{2}\right)=\operatorname{Pf}\left(\frac{f\left(z_{i}, z_{j} ; w_{1}, w_{2}\right)}{z_{i}-z_{j}}\right) \tilde{\Psi}_{\mathrm{LJ}}^{q}
$$

where $f\left(z_{i}, z_{j} ; w_{1}, w_{2}\right)=\left(z_{i}-w_{1}\right)\left(z_{j}-w_{2}\right)+\left(z_{i}-w_{2}\right)\left(z_{j}-\right.$ $\left.w_{1}\right)$. It can be interpreted as the insertion of two spin fields $\sigma(w)$ :

$$
\begin{aligned}
\tilde{\Psi}_{\mathrm{Pf}+2 \mathrm{qh}} \propto & \left\langle\psi\left(z_{1}\right) \cdots \psi\left(z_{N}\right) \sigma\left(w_{1}\right) \sigma\left(w_{2}\right)\right\rangle \\
& \times \tilde{\Psi}_{\mathrm{LJ}}^{q} \prod_{i}\left(z_{i}-w_{1}\right)^{1 / 2}\left(z_{i}-w_{2}\right)^{1 / 2} .
\end{aligned}
$$

The spin fields induce square-root branch singularities in the fermi fields, described by the ope's 22]

$$
\psi(z) \sigma(w) \sim(z-w)^{-1 / 2} \sigma(w)+\ldots .
$$

The branch singularities are cancelled by the explicit square roots, to ensure that the wavefunctions are singlevalued. This fixes the charge of each quasihole to be $1 / 2 q$. We note 16 that quasiholes can be created only in pairs.

In Ref. [16] it was proposed to extend this by defining wavefunctions for $2 n$ quasiholes by inserting $2 n$ spin fields. Since the ope's, by definition, describe short distance properties of correlators independently of what other fields are present, our argument above implies that the quasihole wavefunctions so obtained will all be zeroenergy eigenstates of the appropriate $H_{3}$ (this can again be seen explicitly in the $n=1$ case above). This is analogous to the Laughlin states, where for a suitable pseudopotential Hamiltonian the ground state and states with quasiholes added are zero-energy states [3]. However, for the present case, there is not just a unique state for each set of quasihole positions. Instead there are $2^{n-1}$ linearlyindependent functions (conformal blocks) of the $z_{i}$ 's for $2 n$ spin fields. This number follows from the ope of the spin fields [22]:

$$
\sigma(z) \sigma(w) \sim(z-w)^{-1 / 8} I+\operatorname{const}(z-w)^{3 / 8} \psi(w)+\ldots
$$

Here the higher-order terms fall into two families since the behaviour of each term as $z \rightarrow w$ differs from that of one of the first two terms by an integer power of $(z-w)$. Then in a correlator containing $\sigma\left(w_{1}\right) \sigma\left(w_{2}\right) \cdots$, as $w_{1} \rightarrow w_{2}, w_{3} \rightarrow w_{4}$, etc we can choose a member of either set of terms for each pair of $\sigma$ 's, which would give $2^{n}$ terms, except that the total number of $\psi$ 's in the correlator must be even, so we get $2^{n-1}$ blocks, for $N$ (the number of $\psi$ insertions) odd or even. In principle, the ope's can be used to fix the actual form of the correlators for $2 n \geq 4$. In practice, it was more convenient to find all the zero-energy states by explicit construction, and it was shown that for fixed positions of the quasiholes, the number of states is $2^{n-1}$ 21, 18. Thus the results for the Pfaffian are in complete agreement with these predictions based on CFT ope's.

These degenerate spaces of quasihole states are the basis for the nonabelian statistics properties of the quasiholes (and similar properties are expected for quasielectrons, or for combinations of quasiholes and quasielectrons). As the locations $w_{i}$ of the spin fields are exchanged by analytic continuation, the conformal blocks are mapped to linear combinations of each other (monodromy) [22]. The conjecture of Ref. [16] was that when the quasiholes are exchanged adiabatically as in Ref. [28], these functions exhibit nonabelian statistics, meaning that the effect on members of the space is a linear transformation, which can be described by a $2^{n-1} \times 2^{n-1}$ matrix by choosing a basis, and that this is the same as the matrix obtained by analytic continuation. This replaces the usual Berry phase that describes ordinary (abelian) fractional statistics; matrices representing dis- 
tinct exchanges do not usually commute, hence the term "nonabelian". The existence of many blocks is thus a necessary but not sufficient condition for nonabelian statistics (the adiabatic exchange has not yet been calculated explicitly) 29.

We now discuss the extension of these results to the parafermion states for $k>2$. In place of the spin fields for the Majorana fermion, the parafermion system has "fields" (chiral vertex operators) $\Phi_{m}^{l}$ 25,26], where $l=0$, $1, \ldots, k$, while $m$ is a periodic variable with period $2 k$, so $m=0,1, \ldots, 2 k-1$, and further $l-m=0(\bmod 2)$. In the ope's of $\Phi_{m}^{l}$, the $m$ 's add $\bmod 2 k$, however, there are also identifications which imply that $\Phi_{k+m}^{k-l}=\Phi_{m}^{l}$ are the same operator, so the values of $m$ can be restricted so as to get each of the $k(k+1) / 2$ distinct fields once; a convenient way to do this is by restricting $-l<m \leq l$. In this notation, $\Phi_{0}^{0}=\Phi_{k}^{k}=I$ and $\Phi_{2 \ell-k}^{k}=\Phi_{2 \ell}^{0}=\psi_{\ell}$. The special cases $\sigma_{l}=\Phi_{l}^{l}, l=1, \ldots, k,\left(\sigma_{k}=I\right)$, are called primary fields for the parafermion algebra. $\sigma_{1}$ in a sense generates the whole set by repeated operator products, and is a natural analogue for $\sigma$, to which it reduces for $k=2$. We propose to insert a number $n k$ of $\sigma_{1}$ 's into the correlator of parafermion currents to obtain the basic quasihole states; these are zero-energy states for our special Hamiltonians by the preceding argument. We use $\sigma_{1}$ because this leads to the minimal charge for the quasiholes. Thus our proposal for quasihole states is to use

$$
\begin{aligned}
& \tilde{\Psi}_{\text {para+qh }}^{(M)}\left(z_{1}, \ldots ; w_{1}, \ldots, w_{n k}\right)= \\
& \left\langle\psi_{1}\left(z_{1}\right) \cdots \psi_{1}\left(z_{N}\right) \sigma_{1}\left(w_{1}\right) \cdots \sigma_{1}\left(w_{n k}\right)\right\rangle \\
& \quad \times \tilde{\Psi}_{\mathrm{LJ}}^{M+2 / k} \prod_{i=1}^{N} \prod_{p=1}^{n k}\left(z_{i}-w_{p}\right)^{1 / k}
\end{aligned}
$$

In these states, $N_{\phi}=\nu^{-1} N-(M+2)+n$. Once again, this will in general define a whole set of states (conformal blocks), not a unique state. In the last factor, the exponent $1 / k$ is chosen to cancel the branch singularity as any $\psi_{1}$ approaches any $\sigma_{1}$, which is determined by the ope,

$$
\psi_{1}(z) \Phi_{1}^{1}(0) \sim z^{-1 / k} \Phi_{3}^{1}(0)+\ldots
$$

(see Refs. 25,26]). This is the weakest singularity for any of the $\Phi_{m}^{l}$, so gives the smallest charge for a quasihole. The charge on the quasiholes is determined entirely by the Laughlin-like part of the wavefunction, not the conformal block of the parafermions, so the usual plasma argument shows that the charge (number of particles missing from the vicinity of $\left.w_{p}\right)$ is $\nu / k=1 /(M k+2)$ (use of the other spin fields $\sigma_{l}$ in general give charges $\nu l / k$, and by including additional Laughlin quasihole factors we can add integer multiples of $\nu$ to these charges). $M k+2$ is the denominator of $\nu=k /(M k+2)$, however $k$ and $M k+2$ have a common factor if and only if $k$ is even, and this factor is just 2 (e.g. for the Pfaffian, $k=2$ ). Therefore, when $k$ is even, the charge is fractionalized compared with the usual value in a Laughlin or hierarchy state for a spin-polarized single component system at the same filling factor, which is always $1 / q$ for filling factor $p / q$, where $p, q$ have no common factors. The next nontrivial example is $k=4$, where with $M=1$ for fermions we obtain a $\nu=2 / 3$ state, or $\nu=1 / 3$ by particle-hole inversion, with charge $\pm 1 / 6$ excitations.

The explicit function for $n=1$, that is $k$ quasiholes, is the same as $\tilde{\Psi}_{\text {para }}^{(0)}$, except that a factor of the form

$$
\Gamma\left(z_{1}, \ldots, z_{k} ; w_{1}, \ldots, w_{k}\right)=\prod_{i}\left(z_{i}-w_{i}\right)
$$

for each cluster of $k$ particles is inserted inside the sum on permutations $P$ in Eq. 2.16); the permutations act only on the $z_{i}$ 's, not the $w_{p}$. This generalizes the function $f$ above for the Pfaffian, except that once again the symmetrization is now done all at once by the sum over $P$, which can still be restricted as before. The resulting function is also symmetric in the $w_{p}$ 's.

For more quasiholes, we do not have the explicit functions in general, but we can count the number of zeroenergy states for fixed positions of the quasiholes in the above construction, using the CFT, in a similar way as for the Pfaffian state above; we will consider $k=3$ explicitly. We require the ope's of the fields $\Phi_{m}^{l}$, which are given in Ref. 26] for all $k$, and will not be written down explicitly here. Using the ope's one finds by repeatedly taking ope's with $\sigma_{1}$, that the number of conformal blocks in the parafermion theory for $k=3$ for $3 n$ quasiholes is a Fibonacci number, $F_{3 n-2}$, where we define $F_{1}=1$, $F_{2}=2, F_{3}=3, F_{4}=5$, and $F_{m}=F_{m-1}+F_{m-2}$ in general [30]. Thus, the number of linearly-independent zero-energy states for our Hamiltonian is also (at least) $F_{3 n-2}$ for fixed positions of $3 n$ quasiholes, in the $k=3$ case, provided $N$ is sufficiently large. For large $n$, this number approaches $\sim(2+\sqrt{5})^{n}$. As $k$ increases, the expressions for the $n$ dependence for the parafermion cases will become progressively more complex than that for the Pfaffian $(k=2)$ case, which was $2^{n-1}$.

The results for the quasiholes can be compared with numerical results for the four-body Hamiltonian. The results for the total number of zero-energy states (compare Ref. [18] for results on the Pfaffian state), were calculated for $N=6,9$, and 12 fermions at $\nu=3 / 5$, but should be independent of $M$. First, the ground state at the stated $N_{\phi}$ is unique, and for small sizes was verified to be given by the explicit polynomial above. For one flux added $(n=1)$, that is 3 quasiholes, the number of states was

$$
\left(\begin{array}{c}
N / 3+3 \\
3
\end{array}\right)
$$

as one would expect for 3 bosons in $N / 3+1$ orbitals, or from the explicit wavefunctions above. For $n=2$ flux added, that is 6 quasiholes, the numbers of states were in agreement with the formula 


$$
\left(\begin{array}{c}
N / 3+6 \\
6
\end{array}\right)+3\left(\begin{array}{c}
N / 3+5 \\
6
\end{array}\right)+\left(\begin{array}{c}
N / 3+4 \\
6
\end{array}\right)
$$

which is similar in form to those found for the Pfaffian state [18. Assuming this works for all larger $N$, and dividing by the first term which is the value of the positional degeneracy that would be expected if the quasiholes obeyed abelian statistics [18], we obtain the ratio 5 in the thermodynamic limit, which agrees with the number expected from conformal field theory. Similarly, for 9 quasiholes $(n=3)$, the results agree with the formula

$$
\left(\begin{array}{c}
N / 3+9 \\
9
\end{array}\right)+10\left(\begin{array}{c}
N / 3+8 \\
9
\end{array}\right)+10\left(\begin{array}{c}
N / 3+7 \\
9
\end{array}\right)
$$

and so we expect the ratio to be 21 in the thermodynamic limit, as expected for fixed positions of the quasiholes. For more quasiholes, because of size limitations we have not been able to obtain any such formulas. Thus we find a satisfying agreement with our prediction, which tends to confirm that all the zero-energy quasihole states are obtained by inserting $\sigma_{1}$ 's in the parafermion correlator.

We have not obtained results for the 4-body Hamiltonian ground states on the torus, or the edge excitations (compare [20,18]), either analytically or numerically. However, we expect that these calculations would lead in general to the conclusion that the number of sectors of edge states, or ground states on the torus, is $(k+1)(M k+2) / 2$ (notice that this integer is divisible by the denominator of the filling factor in all cases, as required by Ref. [31]). This result is based on a natural structure for the CFT, including the U(1) charge sector as well as the parafermion sector. Our analysis of this theory, into which we will not go in detail here, also indicates that while for $k=2$, there are neutral fermion excitations, both at non-zero energy in the bulk and as gapless excitations at the edge [16,17,20], for $k>2$ the analogous parafermion $\psi_{1}$ excitations carry charge $2 \nu / k$ plus multiples of $\nu$. The excitation containing a $\psi_{1}$ and charge 1 is identified with the physical hole, as usual, and in the CFT interpretation that applies (for example) to the CFT of edge excitations, it generates the chiral algebra, as in the Pfaffian case [16.20]. There are, however, neutral parafermion excitations for $k$ even, that contain $\psi_{k / 2}$. These can be viewed as being made from $k / 2$ particles and $(M k+2) / 2$ flux. These are simply fermions for $k / 2$ odd, bosons for $k / 2$ even, and are like the usual composite particles. Also, for all $k$, there are nontrivial neutral excitations originating from the spin fields, $\Phi_{0}^{l}=\Phi_{k}^{k-l}$ in the earlier notation. For $k=3$, the latter, $\Phi_{0}^{2}$, is the only field other than the parafermions $\psi_{\ell}$ and the spin fields $\sigma_{l}$. These have no analogues either in the hierarchy or the Pfaffian $(k=2)$ states, though they do in the generalized hierarchy states, such as the 331 state [20]. We further note some isomorphisms of the algebraic structures. For $M=0$, the full chiral algebra, including the $\mathrm{U}(1)$ charge sector, is the level $k \mathrm{SU}(2)$
Kac-Moody current algebra, with representations (sectors) labelled by "spin" $j=l / 2=0,1 / 2, \ldots, k / 2$ (this spin is of course not the physical spin, which is always polarized). For $M=1$, the chiral algebra is the so-called $N=2$ superconformal algebra in the antiperiodic sector, and the representations for each $k$ make up the known discrete series $k=1,2, \ldots$, for this algebra [32]. In these cases, we have simply recovered known constructions of these algebras and representations from the parafermions 25.33. The special cases $k=1$ (the Laughlin state) and $k=2$ (the Pfaffian state) of these were mentioned earlier 116,20, and the $\mathrm{SU}(2) k=2, M=0$ case was used in a recent paper [34].

\section{COULOMB INTERACTION IN THE FIRST EXCITED LANDAU LEVEL}

We next turn to finite-size calculations. We have numerically constructed the wavefunctions of parafermion states for $\nu=3 / 5(k=3, M=1)$, and $\nu=2 / 3(k=4$, $M=1$ ) for small sizes on the sphere and have confirmed that the $k+1$-body Hamiltonians possess unique zeroenergy ground states at the given flux $N_{\phi}$. For $k=3$, we have also obtained the excitation spectrum, both for the model $k+1$-body interaction and the Coulomb potential in the $\mathcal{N}=1$ Landau level, and have studied the overlaps of the Coulomb ground state with our state as well as with the usual hierarchy states as the pseudopotential $V_{1}$ is varied about the its $(\mathcal{N}=1)$ Coulombic value. Below we describe these results.

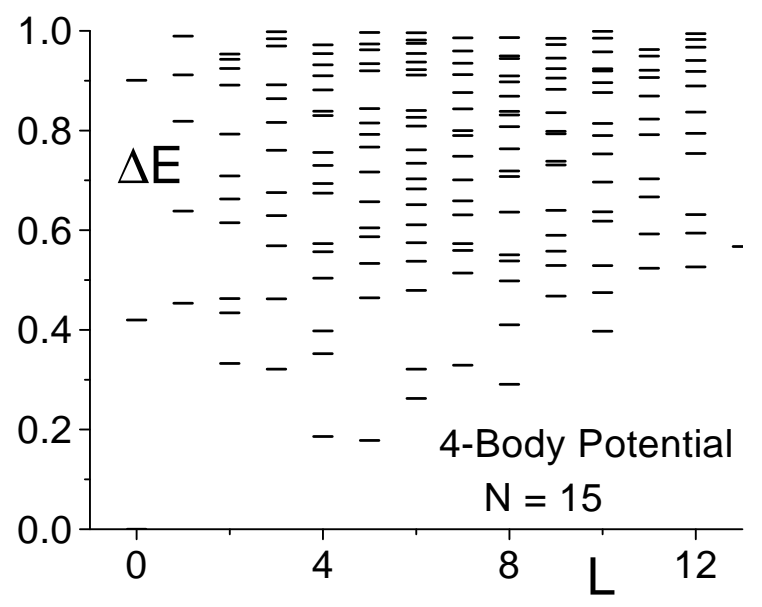

FIG. 1. The low-lying spectrum for the four-body Hamiltonian for $N=15$ electrons. The ground state is at $L=0$, $\Delta E=0$.

Fig. 1 shows the low-lying spectrum for $N=15$ and $\nu=3 / 5$ for the four-body Hamiltonian. The ground state is at $L=0$, zero energy. The low-lying spectrum bears 
some resemblance to the "hanging chain" shape seen in paired systems.

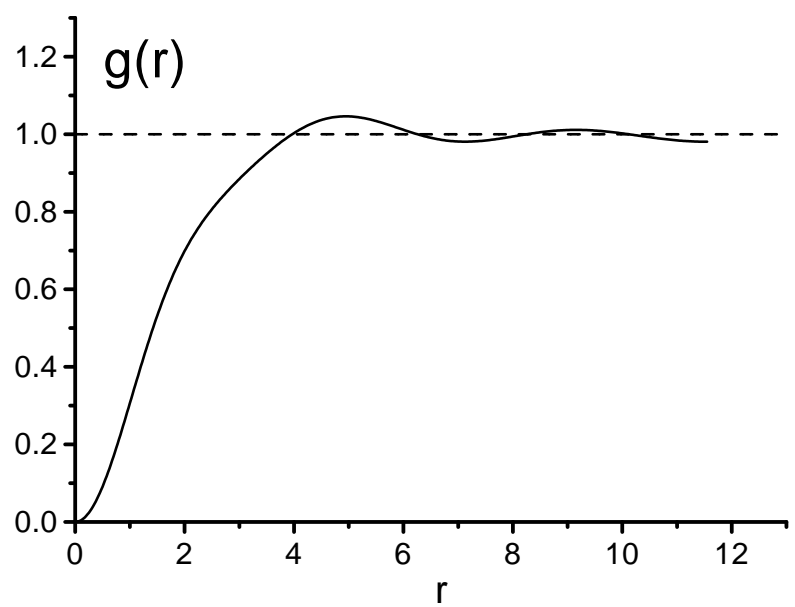

FIG. 2. Pair correlation function of the $\nu=3 / 5$ parafermion state for $N=18$ electrons on the sphere. $r$ is the great-circle distance.

In Fig. 2 we show the pair correlation function for this same state but for the $N=18$ size system, plotted as a LLL wavefunction, which describes the correlations of the guiding-center coordinates of the particles. Again, the "shoulder" at small $r$ appears to be a characteristic of states in which the particles form clusters, such as pairs, and is also present for the Pfaffian state [18,35]. In addition, the large-distance oscillations are strongly damped. This feature indicates that the state is incompressible.

We have also compared these states with the ground state obtained by diagonalizing the Coulomb potential for the $\mathcal{N}=1$ Landau level. In the LLL, the hierarchy states are most stable and these new ones will not be competitive. The first excited Landau level may be quite a different matter since $V_{1}$ is reduced compared to $V_{3}$ (see e.g. Ref. [3]). Some typical values for $V_{1}, V_{3}$, and $V_{5}$ at $N_{\phi}=22$ are $\mathcal{N}=0: 0.4681,0.2998,0.2422$, and $\mathcal{N}=1: 0.4716,0.3711,0.2800$. For the $\mathcal{N}=1$ Coulomb interaction ground state, the hierarchy state has small overlap-squared, whereas parafermionic states have very large ones. (The hierarchy state was obtained as the ground state of a model pseudopotential consisting only of non-zero $V_{1}$. We caution that these two states occur at different values of $N_{\phi}$ because of the finite shifts on the sphere $\left[N_{\phi}=5 N / 3+1\right.$ for the hierarchy].) For the $k=3$ parafermion state we find $97 \%$ for $N=15$ (where there are 36 states in the $L=0$ Hilbert space) and $88 \%$ for $N=18$ (319L=0 states), compared to at best 1 or $2 \%$ for the hierarchy $3 / 5$ state. This is noteworthy since for these sizes we are very close to a Laughlin $2 / 3$ state (i.e. the Laughlin 1/3 state of holes), because of the finite shifts in the $N-N_{\phi}$ relations on the sphere. For $N=15$ the $N_{\phi}$ for our state coincides with that of a single quasiparticle excitation of the $\nu=2 / 3$ fluid while for $N=18$ it is at the same flux as the $2 / 3$ condensate itself. This is a clear disadvantage for the parafermion states particularly if we try to vary the short-range component of the Haldane pseudopotential $V_{1}$. Not surprisingly, increasing $V_{1}$ by a few percent seems to favor the Laughlin state. However, one would expect the hierarchy $\nu=3 / 5$ state, against which our state will be ultimately competing for large sizes, to show a slower rate of stabilization upon increasing $V_{1}$ away from its second Landau level value.

To study this issue we compare the overlap-squared of the ground state of $H=H_{\mathrm{Coul}, \mathcal{N}=1}+\delta V_{1}$ with both our state and the hierarchy $3 / 5$ state. Fig. 3 shows these overlaps as a function of $\delta V_{1}$ for the $N=12(52 L=0$ states) hierarchy and $N=15$ parafermion state. (The sharp drop in the parafermion curve at $\delta V_{1}$ about 0.03 is due to a level crossing: for larger $\delta V_{1}$, the ground state has $L=2$.) It appears that our state remains stable for increases of $\delta V_{1}$ of up to $7-8 \%$ from the Coulomb value. Note however that the hierarchy state is not fully stabilized until $V_{1}$ is increased by $20 \%$ of its Coulomb value. For large sizes there will be less interference from $\nu=2 / 3$ and the stability domain for our state may extend well beyond $7-8 \%$.

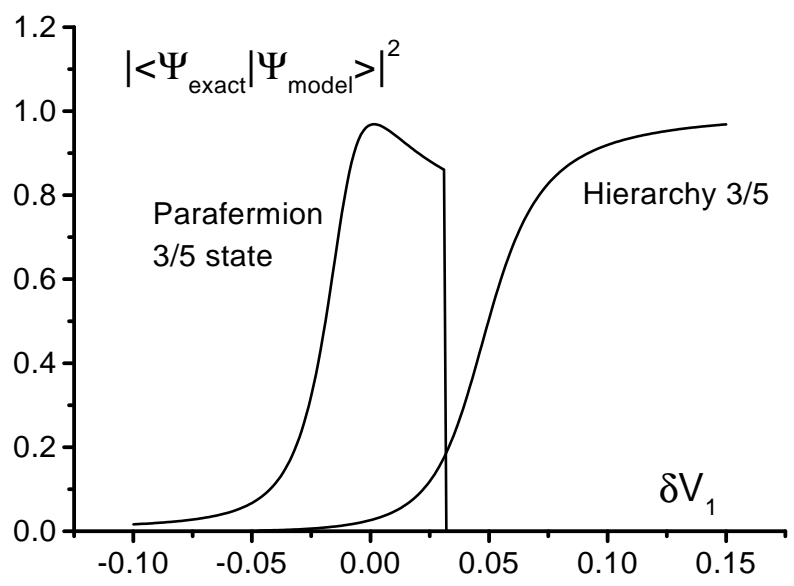

FIG. 3. Overlap-squared of the two reference states, the $\nu=3 / 5$ parafermion state $(N=15)$ and the $\nu=3 / 5$ hierarchy state $(N=12)$, with the state obtained by diagonalizing the $\mathcal{N}=1$ Coulomb potential with an added $\delta V_{1}$ component.

Finally, in Fig. A we show the low-lying spectrum for the pure Coulomb case for $N=15$. Again one finds some similarity with that of the four-body Hamiltonian (Fig. 11), although in neither case is there a clear gap to a continuum of excited states. We defer precise gap estimations incorporating finite layer thickness and other effects that modify the gap values, as well as detailed 
studies of the quasiparticles, to future work.

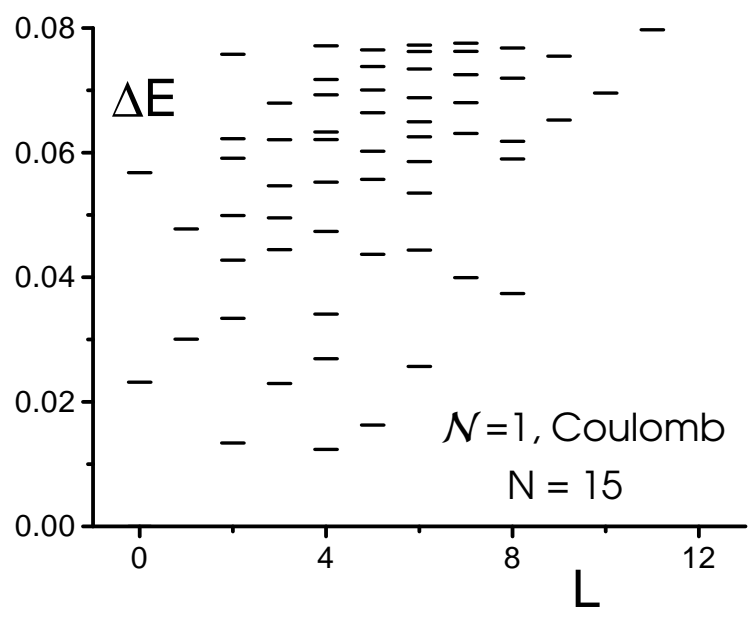

FIG. 4. Same as Fig. 1 but for the $\mathcal{N}=1$ Coulomb potential. The ground-state energy has been subtracted in the spectrum.

\section{CONCLUSION}

To conclude, we have obtained the ground-state wavefunctions for the $k+1$-body Hamiltonians, and for the case $k=3$, we have counted quasihole states, and (for $M=1$ ) found excellent overlaps with the ground state of the Coulomb interaction for spin-polarized electrons in the first excited $(\mathcal{N}=1)$ LL, at total filling factor $\nu=2+3 / 5$; by a particle-hole transformation, this also applies at $\nu=2+2 / 5$.

Our parafermion states contain clusters of $k$ particles. For the Pfaffian state $(k=2)$ at $\nu=1 / 2$, it has been suggested 13, 14 that it may be favored in the $\mathcal{N}=1 \mathrm{LL}$, because of this feature. That is, the correlation hole around each particle that is obtained by the Laughlin-style correlations, as a result of attaching $q$ vortices to each particle (for filling factor of the topmost LL equal to $1 / q$ ) [36], may not be sufficient to obtain the lowest energy in higher LLs, because of the "form factor" associated with these LLs - the real space wavefunction does not vanish when particles coincide [13]. However, by forming a cluster of two or more particles, the energy gained from the larger correlation hole surrounding the cluster may outweigh that lost within the cluster itself. Very similar physics is suggested by recent work motivated by the form of the pseudopotential interaction in higher LLs; the latter are also a consequence of the form factors. These works consider the formation of crystalline phases in which the particles are clustered, so there is more than one per unit cell [37]. If this idea is correct, then liquids containing clusters might also be expected to occur in higher LLs, possibly as intermediate phases between the crys- tals and the usual hierarchy states (if we consider varying the short-range part of the interaction away from its physical value, at fixed $\nu$ ). As the LL index $\mathcal{N}$ increases, larger clusters with $k \sim \mathcal{N}$ are expected to be favored 37. It is very interesting that recent experimental work 38 has observed highly-resistive, highly-anisotropic behavior, with nonlinear current-voltage characteristics, at total filling factors greater than 4 , that is in the range $\mathcal{N}=2$ to about 6 . This behavior, seen around the center of each LL for each spin component, appears consistent in principle with the notion of a uniaxial or "striped" crystal phase, which was also predicted in Ref. 37 in this region (the triaxial crystals of clusters were predicted for $\nu$ away from $1 / 2$ ), and thus may support these physical pictures. There is clearly much still to be done to understand the physics in this regime, in which we hope that the parafermion liquid states will play a role.

\section{ACKNOWLEDGMENTS}

We thank A. Ludwig, G. Moore, R.H. Morf, and K. Schoutens for helpful discussions. We also thank the Institute for Theoretical Physics, UCSB, program "Disorder and Interactions in Quantum Hall and Mesoscopic Systems" for a stimulating environment in which this work was completed. This work was supported by NSF grants, nos. DMR-9157484 (NR), DMR-9420560 (ER), and at the ITP by NSF-PHY94-07194. ER is also grateful to ITP for an ITP Scholar award.

[1] For a review, see, e.g., The Quantum Hall Effect, edited by R.E. Prange and S.M. Girvin (Second Edition, Springer-Verlag, New York, 1990).

[2] R.B. Laughlin, Phys. Rev. Lett. 50, 1395 (1983).

[3] F.D.M. Haldane, Phys. Rev. Lett. 51, 605 (1983) and in Ref. [1].

[4] B.I. Halperin, Phys. Rev. Lett. 52, 1583 (1984).

[5] J.K. Jain, Phys. Rev. Lett. 63, 199 (1989).

[6] F.D.M. Haldane and E.H. Rezayi, Phys. Rev. Lett. 60, 956, 1886 (E) (1988).

[7] B.I. Halperin, P.A. Lee, and N. Read, Phys. Rev. B 47, 7312 (1993).

[8] E. Rezayi and N. Read, Phys. Rev. Lett. 72, 900 (1994).

[9] N. Read, Phys. Rev. Lett. 65, 1502 (1990).

[10] B. Blok and X.-G. Wen, Phys. Rev. B (1990).

[11] E. Rezayi, Phys. Rev. B. 36, 5454 (1987); ibid. 43, 5944 (1991); S. Sondhi, A. Karlhede, S.A. Kivelson, and E. Rezayi, Phys. Rev. B 47, 16419 (1993).

[12] R.L. Willett, J.P. Eisenstein, H.L. Störmer, D.C. Tsui, A.C. Gossard, and J.H. English, Phys. Rev. Lett. 59, 1776 (1987). 
[13] R.H. Morf, Phys. Rev. Lett. 80, 1505 (1998), and private communication.

[14] E.H. Rezayi and F.D.M. Haldane, "Transition to paired Hall states in half-filled Landau levels", 1998 APS March Meeting abstracts; http://www.aps.org/BAPSMAR98/abs/S3470.html\#SQ31.001

[15] B.I. Halperin, Helv. Phys. Acta, 56, 75 (1983).

[16] G. Moore and N. Read, Nucl. Phys. B360, 362 (1991). See also N. Read and G. Moore, Prog. Theor. Phys. (Kyoto) Supp. 107, 157 (1992).

[17] M. Greiter, X.-G. Wen and F. Wilczek, Phys. Rev. Lett. 66, 3205 (1991); Nucl. Phys. B374, 567 (1992).

[18] N. Read and E. Rezayi, Phys. Rev. B 54, 16864 (1996).

[19] X.-G. Wen, Phys. Rev. Lett. 70, 355 (1993).

[20] M. Milovanović and N. Read, Phys. Rev. B 53, 13559 (1996).

[21] C. Nayak and F. Wilczek, Nucl. Phys. B479, 529 (1996).

[22] A. Belavin, A. Polyakov, and A. Zamolodchikov, Nucl. Phys. B241, 33 (1984).

[23] N. Read and E. Rezayi, Yale/Calstate report, 1993, unpublished.

[24] A sum over permutations within a triple $i, j, k$ is not required.

[25] A.B. Zamolodchikov and V.A. Fateev, Sov. Phys. JETP 62, 215 (1985).

[26] D. Gepner and Z. Qiu, Nucl. Phys. B 285 [FS19], 423 (1987).

[27] X.-G. Wen and Y.-S. Wu, Nucl. Phys. B419 [FS], 455 (1994); X.-G. Wen, Y.-S. Wu and Y. Hatsugai, Nucl. Phys. B422 [FS], 476 (1994).
[28] D. Arovas, J.R. Schrieffer, and F. Wilczek, Phys. Rev. Lett. 53, 722 (1984).

[29] In some cases studied in Ref. 18], the number of zeroenergy states was greater than the number of conformal blocks. In any case, the argument given here does not establish completeness, so the number of conformal blocks should be viewed as a lower bound on the degeneracy. However, in the present case for general $k$ values, we expect the number of zero energy states to saturate this bound.

[30] This result was pointed out to us by K. Schoutens, private communication.

[31] F.D.M. Haldane, Phys. Rev. Lett. 55, 2095 (1985).

[32] W. Boucher, D. Friedan, and A. Kent, Phys. Lett. B 172, 316 (1986).

[33] A. B. Zamolodchikov and V.A. Fateev, Sov. Phys. JETP 63, 913 (1986).

[34] E. Fradkin, C. Nayak, A. Tsvelik, and F. Wilczek, condmat/9711087.

[35] K. Park, V. Melik-Alaverdian, N.E. Bonesteel, and J.K. Jain, cond-mat/9806271.

[36] N. Read, Semicond. Sci. Technol. 9, 1859 (1994) condmat/9501090.

[37] A.A. Koulakov, M.M. Fogler, and B.I. Shklovski, Phys. Rev. Lett. 76, 499 (1996); Phys. Rev. B 54, 1853 (1996); R. Moessner and J.T. Chalker, Phys. Rev. B 54, 5006 (1996).

[38] M.P. Lilly, K.B. Cooper, J.P. Eisenstein, L.N. Pfeiffer, and K.W. West, cond-mat/9808227. 